\title{
HERBICIDAS ALACHLOR, PENDIMETHALIN E TRIFLURALIN NA NODULAÇÃO E CRESCIMENTO INICIAL DE PLANTAS DE AMENDOIM
}

\author{
Maria de Fátima da S. P. Peixoto ${ }^{1}$, Luciano S. de V. Sampaio ${ }^{2}$, Clóvis P. Peixoto ${ }^{1}$, Heraldo S. de V. \\ Sampaio $^{2}$, Regilene Angélica da S. Souza ${ }^{3}$, Carla Gabriela S. Meneses ${ }^{4}$ \\ e Jorge Gabriel M. N. de Jesus ${ }^{4}$
}

\footnotetext{
${ }^{1}$ Eng $^{\circ}$. Agrônomo, Dr., Professor. Escola de Agronomia da Universidade Federal da Bahia/ Departamento de Química. Caixa Postal 82. Cruz das Almas, BA 44380-000 fpeixoto@ufba.br.

${ }^{2}$ Eng $^{\circ}$. Agrônomo, M. Sc., Professor. Escola de Agronomia da Universidade Federal da Bahia/ Departamento de Fitotecnia.

${ }^{3}$ Estudante de Graduação, Bolsista do PET. Escola de Agronomia da Universidade Federal da Bahia.

${ }^{4}$ Eng $^{\circ}$. Agrônomo. Escola de Agronomia da Universidade Federal da Bahia.
}

\section{RESUMO}

Este trabalho teve como objetivos avaliar o efeito dos herbicidas alachlor, pendimethalin e trifluralin no crescimento inicial de plantas de amendoim (Arachis hypogaea L.) em casa de vegetação, por um período de trinta dias, e o número e massa seca dos nódulos após 60 dias do plantio. O experimento foi conduzido na casa de vegetação do Departamento de Química Agrícola e Solos da Escola de Agronomia da Universidade Federal da Bahia. O delineamento experimental do primeiro ensaio foi inteiramente casualizado, com quatro tratamentos e três repetições. Os tratamentos utilizados foram: alachlor (2,90 kg ha); pendimethalin $(1,00 \mathrm{~kg}$ ha), trifluralin $(0,89 \mathrm{~kg}$ ha) e testemunha. As parcelas foram constituidas de bandejas de plástico de 70 $\mathrm{cm}$ x $50 \mathrm{~cm}$ x $10 \mathrm{~cm}$, tendo como substrato um solo Latossolo amarelo álico coeso, utilizando-se sementes da variedade BRS 151L7, oriundas da EMBRAPA/CNPA. No segundo ensaio, a unidade experimental constituiu-se de vasos plásticos de $5 \mathrm{~kg}$ com os mesmos tratamentos, substrato e delineamento experimental, com oito repetições. Avaliou-se os seguintes parâmetros: percentagem de emergência, plântulas anormais, sementes mortas, altura da planta, comprimento da raiz, índice de velocidade de emergência, massa seca da parte aérea e da raiz, número e massa seca de nódulos. Os herbicidas testados, apesar de estimularem a nodulação inicial das plantas de amendoim, reduzem a porcentagem de emergência, massa seca da parte aérea e altura das plantas, devendo ser recomendados com restrições nas condições estudadas.

Palavras-chave: fixação biológica, nitrogênio, qualidade fisiológica, semente.

\section{ABSTRACT \\ Herbicides alachlor pendimethalin and trifluralin on the nodulation and initial growth of peanut plants}

This work had the objective of evaluating the effect of the herbicides alachlor, pendimethalin and trifluralin on the initial growth of peanut plants (Arachis hypogaea L.) under greenhouse conditions for a period of thirty days as well as nodule number and dry weight for sixty days after the sowing period. The experiment was conducted at the Agronomy College of the Federal University of Bahia. The first experimental design was completely randomized with four treatments (control - rate zero; alachlor $-2.90 \mathrm{~kg} / \mathrm{ha}$, pendimethalin $-1.00 \mathrm{~kg} / \mathrm{ha}$ and trifluralin $-0.89 \mathrm{~kg} / \mathrm{ha}$ ) and three replications. For each replication, plastic trays $(70 \mathrm{~cm} \times 50 \mathrm{~cm} \times 10 \mathrm{~cm}$ ) were used with a yellow Latosol as the substrate and peanut seeds of the variety BRS 151-L7 provided by EMBRAPA-CNPA. A second experiment was conducted with plastic pots of $5 \mathrm{~kg}$, the same substrate, same treatments and experimental design with eight replications. The following parameters were evaluated: seedling emergence, percentage of abnormal seedlings and dead seeds, plant height, index of seedling emergence velocity, root and shoot dry weight,number and dry weight of nodules. The results show that herbicides stimulate the initial nodulation of peanut plants, but reduce the percentage of emergence, shoot dry weight and plant height. Therefore, the herbicides should be recommended with restrictions, for the studied conditions.

Key words: biological fixation, nitrogen, physiological quality, seeds. 


\section{INTRODUÇÃO}

O amendoim (Arachis hypogaea L.) é uma planta dicotiledônea, da família Leguminosae, originária do Brasil, e bastante cultivada no Recôncavo Baiano. Durante as fases iniciais do seu desenvolvimento sofre a competição das plantas daninhas que prejudicam sensivelmente a produção (Sader et al., 1979).

O controle químico das plantas daninhas na cultura do amendoim iniciou-se a partir de 1950, com herbicidas seletivos tais como o dinoseb. Na década de 60 , com o desenvolvimento dos herbicidas do grupo das dinitroanilinas, surgiu o trifluralin. Os herbicidas do grupo cloroacetanilidas, tais como alachlor e metalachlor, foram em seguida incorporados aos herbicidas disponíveis para o uso na cultura do amendoim (Bridges et al., 1984).

Várias pesquisas têm sido feitas com o objetivo de verificar o que ocorre com os herbicidas no solo após sua aplicação. Diversos trabalhos tem relatado sua degradação e persistência (Stolp \& Shea, 1995; Monteiro, 1997; Vanderheyden et al.,1997; Yassir et al., 1998), potencial para formação de resíduos (Wais et al., 1995; Barriuso \& Koskinen, 1996; Queiroz, 1997), e biodisponibilidade para plantas (Khan \& Behki, 1990). Na Bahia, especialmente na região do Recôncavo, as pesquisas desenvolvidas quanto ao efeito desses herbicidas sobre alguns parâmetros fisiológicos da semente, nodulação e crescimento inicial das plantas são praticamente inexistentes.

Dentre os herbicidas utilizados na cultura do amendoim cita-se o alachlor, bentazon, lactofen, linuron, pendimethalin e trifluralin. O alachlor pertence ao grupo químico das cloroacetamidas, controla algumas mono e dicotiledôneas e é indicado para as culturas de algodão, amendoim, café, canade-açúcar, milho e soja. Impedem o crescimento e o desenvolvimento da parte aérea das plantas, sendo que a absorção ocorre pelo coleóptilo nas gramíneas e pelo epicótilo ou hipocótilo nas dicotiledôneas, durante o trajeto pela zona do solo onde se encontra o produto. Interfere com a atividade da giberelina e/ou com a elongação de lipídios na célula. Sem a giberelina, a enzima alfa amilase não é produzida na camada de aleurona e não há decomposição do amido em glicose no endosperma, ocorrendo falta de energia para o desenvolvimento das plântulas, após a germinação da semente. Por outro lado, a falta de lipídios de cadeia muito longa nas células da folhagem nova, impede a formação de ácidos graxos e de cera cuticular, diminuindo a proteção das folhas contra as perdas de água. A conseqüência final desses processos em espécies suscetíveis é o atraso da elongação e da divisão celular, o que finalmente leva a planta à morte (Vidal \& Fleck, 2001a).

O pendimethalin e o trifluralin pertencem ao grupo químico das dinitroanilinas e controlam predominantemente gramíneas anuais e algumas dicotiledôneas anuais. São indicados para as culturas do amendoim, algodão, alho, arroz, café, milho, soja, entre outras. São absorvidos a partir do solo através das raízes e da parte aérea que se desenvolvem em contato com o solo tratado. Em função de serem imóveis na planta, a atividade herbicida é observada principalmente nas raízes. Esses herbicidas unem-se fortemente à tubulina, proteína que faz parte dos microtúbulos, responsáveis pela movimentação dos cromossomos durante a divisão celular. Consequentemente, não há movimentação dos cromossomos e a divisão celular é interrompida na prófase. Os meristemas das raízes ficam atrofiados e as plântulas apresentam reduzida capacidade de absorção de água e nutrientes e acabam morrendo antes de emergirem do solo. As que conseguem emergir podem apresentar caule "inchado" na região de contato com o solo (Vidal \& Fleck, 2001b).

Sader et al. (1979) avaliaram a influência de diferentes doses de trifluralin $(0,1,2,1,6,2,0,2,4$ e 2,8 L/ha do produto comercial), em condições de campo, no controle de plantas daninhas, produção e poder germinativo da semente de amendoim. Concluíram que não houve efeito prejudicial, mesmo nas doses mais elevadas, sobre o poder germinativo da semente, chegando a obter percentagens de germinação de $92 \%$ quando foi utilizada a dose mais elevada. Também não foram notados, em algum dos tratamentos, sintomas de toxicidade à cultura.

Amaral \& Ribeiro (1982) avaliaram a influência dos herbicidas butachlor, pendimethalin e oxadiazon na emergência de plântulas de arroz (utilizaram o índice de velocidade média de germinação) em um ensaio em casa de vegetação, nas doses recomendadas pelo fabricante. Concluiram que o efeito dos herbicidas na emergência das plântulas variou de acordo com cada produto em particular. Os herbicidas pendimethalin e butachlor retardaram a emergência e reduziram o peso da matéria seca aos 28 dias após a semeadura, mas não afetaram a produção final de grãos. Constataram também alguns sintomas tóxicos durante a emergência das plântulas. $\mathrm{O}$ pendimethalin caracterizou-se por provocar um engrossamento na região do colo de muitas plântulas emergentes, especificamente junto ao nó do coleóptilo, impossibilitando a formação e/ou crescimento normal de raízes adventíceas; as folhas primordiais dessas plantas injuriadas, em grande parte tornaram-se encarquilhadas, sem condições de se desenvolverem ou de prosseguirem o desenvolvimento de forma normal.

Em trabalho realizado com a cultura do amendoim, avaliando-se a fitotoxicidade do herbicida flumetsulam, aplicado em pré-plantio e incorporado ao solo, verificou-se que aplicações de doses acima de $0,14 \mathrm{~kg} / \mathrm{ha}$ causavam injúrias visíveis (Johnson \& Mullinix, 1996).

Novo \& Ortolan (1991) estudaram a influencia da nodulação espontânea de plantas de amendoim em rotação com cana-de-açúcar em várias propriedades que utilizaram herbicidas e avaliaram a tolerância do rizóbio isolado do solo à trifluralina. Observaram que $100 \%$ das plantas nodularam e o Bradyrizobium nativo associado ao amendoim apresentou alta tolerância a trifluralina (lastro de 640 ppm) em meio de cultura. 
Rezende et al. (1985) em estudo realizado para avaliar a influência da aplicação dos herbicidas fluorodifen $(0,9 \mathrm{~kg} / \mathrm{ha})$, pendimethalin $(0,75 \mathrm{~kg} / \mathrm{ha})$, fluordifen + pendimethalin $(0,45+$ $0,37 \mathrm{~kg} / \mathrm{ha})$, chloramben $(1,91 \mathrm{~kg} / \mathrm{ha})$, trifluralin $(0,97 \mathrm{~kg} / \mathrm{ha})$ e chloramben + alachlor $(0,95+0,66 \mathrm{~kg} / \mathrm{ha})$ no rendimento de grãos, nodulação e qualidade fisiológica de sementes de soja, concluiram que a nodulação, a percentagem de germinação, o vigor e a população final não foram influenciados pelos herbicidas testados.

Peixoto et al. (2002), avaliando o efeito do herbicida trifluralin na nodulação e qualidade fisiológica de plantas de soja, verificaram que na dose de 1,5 L/ha este herbicida não afetou a germinação; no entanto, em todas as doses utilizadas prejudicou a nodulação e promoveu o decréscimo da massa seca da parte aérea e da raiz.

Em função da utilização destes herbicidas em práticas agrícolas no cultivo do amendoim, da representatividade deste para a região do Recôncavo Baiano e da importância de se conhecer os efeitos dessas moléculas organo-sintéticas na nodulação e qualidade fisiológica das plantas, aliado à relativa escassez de informações nesta área, objetiva-se neste estudo verificar o efeito dos herbicidas alachlor, pendimethalim e trifluralin na nodulação e crescimento inical de plantas de amendoim.

\section{MATERIAL E MÉTODOS}

Dois ensaios foram conduzidos na casa de vegetação do Departamento de Química Agrícola e Solos da Escola de Agronomia da Universidade Federal da Bahia, em Cruz das Almas, Bahia.

\section{Primeiro ensaio}

O solo utilizado como substrato foi um Latossolo Amarelo álico coeso, representativo da região do Recôncavo Baiano e pertencente a zona dos tabuleiros costeiros, cujos atributos químicos encontram-se na Tabela 1 . A coleta foi feita com auxílio de um trado a $20 \mathrm{~cm}$ de profundidade em uma área não cultivada. Em seguida, os solos foram colocados para secar ao ar e processados para obter-se a terra fina seca ao ar (TFSA). Para manutenção da umidade durante todo o ensaio, determinou-se a capacidade de campo pelo método do torrão separado pela frente de molhamento (TSFM), descrito por Costa (1983).

Os herbicidas utilizados foram alachlor, pendimethalin e trifluralin, nas doses de $5 \mathrm{~L} / \mathrm{ha}, 2 \mathrm{~L} / \mathrm{ha}$ e $2 \mathrm{~L} / \mathrm{ha}$, respectivamente (Rodrigues \& Almeida, 1998). Os produtos comerciais e formulações encontram-se discriminados na Tabela 2. As aplicações do pendimethalin e trifluralin foram feitas em pré-plantio incorporado e o alachlor em pré-emergência. Nos dois sistemas utilizou-se um pulverizador costal manual.

Utilizou-se sementes de amendoim da variedade BRS$151 \mathrm{~L} 7$, oriundas da EMBRAPA-CNPA. O plantio foi feito distribuindo-se as sementes em bandejas de plástico com 70 $\mathrm{cm} \times 50 \mathrm{~cm}$ x $10 \mathrm{~cm}$ em 5 linhas, perfazendo-se um total de 50 sementes/bandeja. As avaliações da percentagem de emergência, plântulas anormais e sementes mortas foram feitas trinta dias após o plantio, de acordo com Brasil (1976), e a altura da planta, em centímetros, trinta dias após a semeadura. Para a avaliação da massa seca da parte aérea e da raiz, as plantas foram cortadas ao nível do solo após trinta dias da semeadura, armazenadas em sacos plásticos e pesadas em balança com aproximação até a casa centesimal. Após a pesagem as plantas foram acondicionadas em sacos de papel e secadas em estufa à temperatura de $65^{\circ} \mathrm{C}$ até atingirem peso constante.

$\mathrm{O}$ índice de velocidade de emergência foi determinado com base na fórmula utilizada por Maguire (1962), fundamentada na emergência média diária, como seja:

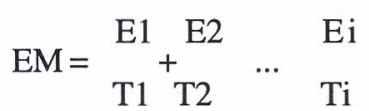

Onde:

$\mathrm{EM}=$ Emergência média diária

E1 até Ei $=$ Percentagem de emergência ocorrida em cada dia $\mathrm{T} 1$ até $\mathrm{Ti}=\mathrm{Tempo}$

Tabela 1. Composição química do Latossolo Amarelo álico coeso dos Tabuleiros Costeiros do Recôncavo baiano. Escola de Agronomia da UFBA, Cruz das Almas, BA. 2002.

\begin{tabular}{|c|c|c|c|c|c|c|c|c|c|c|c|c|}
\hline Prof. (m) & $\mathrm{pH}$ & $\mathrm{P}$ & K & $\mathrm{Ca}+\mathrm{Mg}$ & $\mathrm{Ca}$ & $\mathrm{Mg}$ & $\mathrm{Al}$ & $\mathrm{H}+\mathrm{Al}$ & $\mathrm{Na}$ & $S$ & CTC & $\mathrm{V}$ \\
\hline & $\mathrm{CaCl}_{2}$ & $\mathrm{mg} / \mathrm{kg}$ & & - & 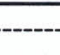 & - & $\mathrm{ol}_{\mathrm{c}} \mathrm{dr}$ & 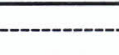 & & ---- & & $\%$ \\
\hline $0,0-0,2$ & 4,4 & 51,0 & 36,0 & 2,3 & 1,5 & 0,8 & 0,1 & 3,3 & 0,8 & 3,2 & 6,5 & 49,0 \\
\hline
\end{tabular}

Tabela 2. Herbicidas utilizados, formulação e doses dos produtos comerciais (p.c.) e dos ingredientes ativos (i.a.). Escola de Agronomia da UFBA, Cruz das Almas, BA. 2002.

\begin{tabular}{|c|c|c|c|c|c|}
\hline \multicolumn{2}{|c|}{ Tratamento } & \multirow{2}{*}{$\begin{array}{c}\text { Formulação e } \\
\text { Conc. (g/L) }\end{array}$} & \multicolumn{3}{|c|}{ Dose } \\
\hline Produto Comercial & Ingrediente Ativo & & L/ha (p.c) & . & kg/ha (i.a) \\
\hline Laço & alachlor & C.E. 480 & 5 & & 2,90 \\
\hline Herbadox & pendimethalin & C.E. 500 & 2 & & 1,00 \\
\hline Trifluralina Defensa & trifluralin & C.E. 445 & 2 & & 0,89 \\
\hline
\end{tabular}




\section{Segundo ensaio}

Utilizou-se como unidade experimental vasos plásti$\cos$ de aproximadamente $5,0 \mathrm{~kg}$ de capacidade. Os tratamentos, substrato e procedimentos para manutenção da umidade foram os mesmos do primeiro ensaio, porém, neste caso utilizou-se oito repetições. Cada vaso recebeu quatro sementes e, após 15 dias efetuou-se o desbaste deixando-se apenas uma planta/vaso. Após 60 dias do plantio as plantas foram retiradas, as raízes separadas e lavadas, realizando-se a contagem dos nódulos, os quais foram colocados em estufa à $65^{\circ} \mathrm{C}$ até apresentarem peso constante, para obtenção da massa seca (Rezende et al., 1985).

\section{Análise Estatística}

Para avaliar os parâmetros do primeiro e segundo ensaios utilizou-se o delineamento experimental inteiramente casualizado, realizando-se análise da variância e comparações entre as médias dos tratamentos pelo teste de Tukey a $5 \%$ de probabilidade.

\section{RESULTADOS E DISCUSSÃO}

O tratamento testemunha foi significativamente superior à todos os demais, indicando que os herbicidas utilizados afetaram a percentagem de emergência (Figura 1), não influenciando, contudo, o seu IVE (Figura 2). Verificou-se também que houve uma variação quanto à intensidade dos efeitos dessas moléculas na emergência, sendo o pendimethalin e o alachlor os herbicidas que mais a prejudicaram (30 e 32\%, respectivamente), e em menor intensidade o trifluralin (55\%) (Figura 1). O pendimethalin e o trifluralin interrompem a divisão celular nos meristemas das raízes causando o seu atrofiamento, prejudicando a absorção de água e nutrientes (Vidal \& Fleck, 2001b). O alachlor é inibidor do crescimento da parte aérea pelo fato de provocar atraso na elongação e divisão celular, o que leva a planta à morte (Vidal \& Fleck, 2001a). Admitindo-se que o comportamento da molécula depende, principalmente, das suas propriedades físico-químicas e tam-

Trifluralina 圆Pendimethalin ‥: Alachlor $\square$ Testemunha

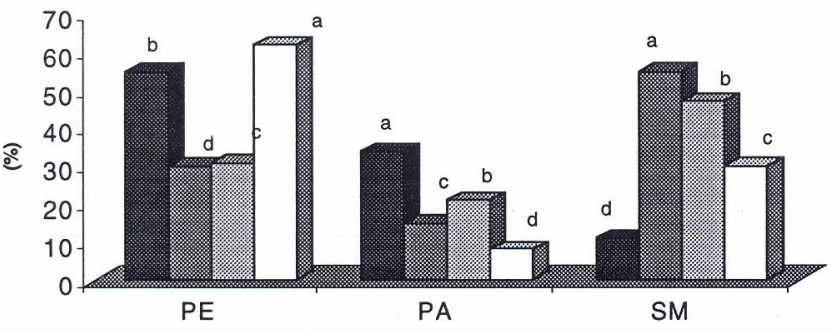

Figura 1- Percentagem de emergência (PE), de plântulas anormais (PA) e de sementes mortas (SM) de amendoim pela ação de herbicidas.

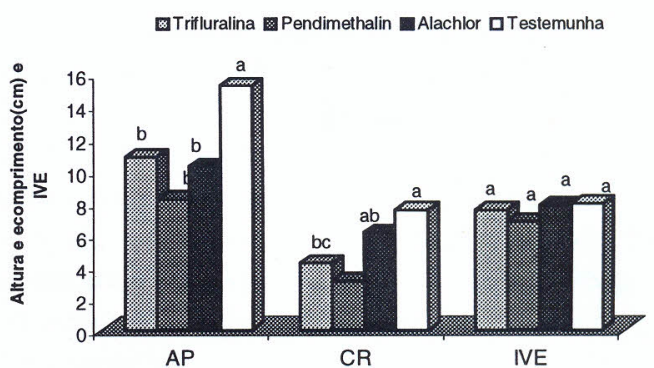

Figura 2 - Altura da planta (AP), comprimento da raiz (CR) e índice de velocidade de emergência (IVE) de amendoim aos trinta dias após a emergência, submetidas a diversos tratamentos com herbicidas.

bém do ambiente, e como o solo utilizado para substrato foi de textura média e baixa CTC (Tabela 1), acredita-se que esses efeitos na percentagem de emergência também podem ser explicados pela baixa adsorção das moléculas pelo solo em questão, permitindo maior concentração na solução do solo e favorecendo assim a absorção. Sader et al. (1979), utilizando trifluralin em amendoim não encontraram efeito prejudicial deste herbicida na germinação; no entanto, Peixoto et al.(2002), trabalhando com soja, verificaram uma diminuição da mesma na dose de 1,5 L/ha. Deve-se levar em conta também, que as sementes de baixo vigor apresentam uma maior permeabilidade das membranas lipo-proteícas, tornando mais efetivo o provável efeito tóxico dos herbicidas.

Quanto a percentagem de plântulas anormais (Figura 1), verificou-se que houve diferença significativa entre os tratamentos e que o herbicida trifluralin foi o que mais provocou injúrias nas plântulas. Em condições de campo, Sader et al. (1979) não encontraram sintoma de toxidade do trifluralin quando testaram diferentes doses desse herbicida na cultura do amendoim; no entanto, segundo Johnson \& Mullinix (1996), herbicidas aplicados em pré-plantio incorporado provocam mais injúrias do que os de pré-emergência.

Em relação à quantidade de sementes mortas (Figura 1), encontrou-se valores de $11 \%, 55 \%, 47 \%$ e $30 \%$, respectivamente, para os herbicidas trifluralin, pendimethalin, alachlor e a testemunha, com diferença significativa entre eles. Verificou-se que o herbicida pendimethalin foi o que apresentou maior percentagem de sementes mortas.

Todos os herbicidas testados afetaram a massa seca acumulada na parte área (MSPA), sendo seus valores significativamente inferiores à testemunha (Tabela 3). A massa da matéria seca das raízes (MSR) variou de 0,46 g/planta no tratamento com pendimethalin, a $1,16 \mathrm{~g} /$ planta no tratamento testemunha, o qual não diferiu do alachlor ( $1,13 \mathrm{~g} / \mathrm{planta})$. O efeito tóxico destas moléculas organo-sintéticas no sistema radicular das plantas depende, entre outros fatores, das suas propriedades fisico-químicas e do modo de absorção. Considerandose que a absorção do pendimethalin é essencialmente radicular e sua translocação muito reduzida, pode-se explicar o menor valor de massa seca das raízes encontrado para esta molécula, devido, provavelmente, a uma maior concen- 
tração da mesma nas raízes. Esse resultado está coerente com o de Amaral \& Ribeiro (1982), onde o pendimethalin causou redução na massa seca das raízes de plantas de arroz aos 28 dias após a semeadura.

Verificou-se que existe uma relação direta entre o comprimento da raiz (Figura 2) e sua massa seca (Figura 3); ou seja, os herbicidas pendimethalin e trifuralin, que reduziram de maneira mais significativa a massa seca, foram os mesmos que contribuiram para o menor comprimento das raízes.

A altura das plantas foi de $8,2 \mathrm{~cm}, 10,2 \mathrm{~cm}$ e $10,8 \mathrm{~cm}$, quando se utilizou pendimethalin, alachlor e trifluralin, respectivamente, não havendo diferença significativa entre os herbicidas testados; no entanto, o tratamento testemunha,
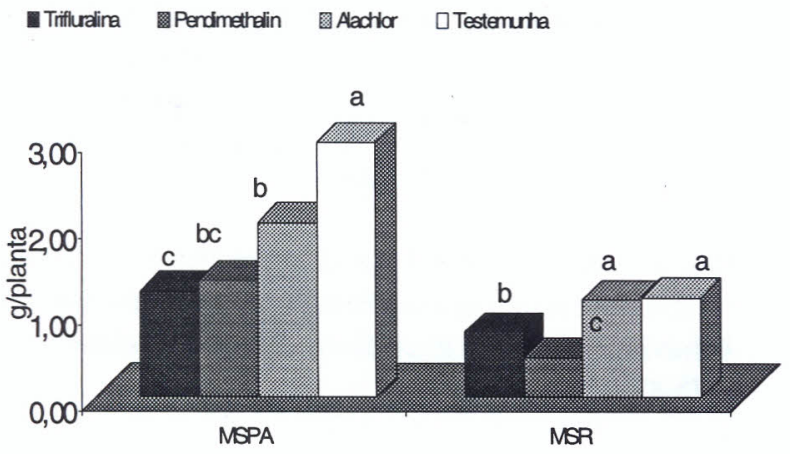

Figura 3- Massa seca acumulada na parte aérea (MSPA) e raiz (MSR) de plantas de amendoim, aos trinta dias após a emergência, submetidas a vários tratamentos com herbicidas.

圆Trifluralina Pendimethalin Alachlor $\square$ Testemunha

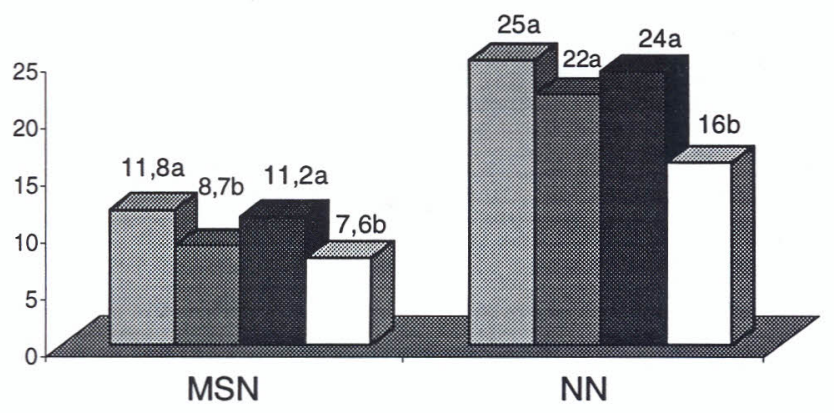

Figura 4 - Massa seca dos nódulos (MSN) (g/planta) e número de nódulos (NN) (n\%planta) de plantas de amendoim, aos trinta dias após a emergência, submetidas a diferentes tratamentos com herbicidas.

com $15,2 \mathrm{~cm}$, foi superior a todos os demais, demonstrando que os herbicidas em questão afetaram negativamente o crescimento inicial da planta (Figura 2).

De maneira geral os herbicidas testados estimularam a nodulação inicial das plantas de amendoim (Figura 4). O tratamento testemunha foi o que apresentou menor número de nódulos (16) e menor massa seca (7,6 g/planta). Consideran- do que não foi feita a inoculação das sementes com rizóbio, a pequena quantidade de nódulos encontrada pode ser explicada pela baixa eficiência dos rizóbios nativos.

\section{CONCLUSÕES}

Os herbicidas trifluralin, pendimethalin e alachlor, apesar de estimularem a nodulação inicial das plantas de amendoim, reduzem a percentagem de emergência, massa seca da parte aérea e altura das plantas, devendo ser recomendados com restrições nas condições estudadas.

\section{LITERATURA CITADA}

AMARAL, A.S. RIBEIRO, A.S. Efeitos de herbicidas e de fungicidas na emergência de plântulas de arroz. Revista Brasileira de Sementes, v.4, n.2, p.33-43, 1982.

BARRIUSO, E.; KOSKINEN, W.C. Incorporating nonextractable atrazine residues into soil size fractions as a function of time. Soil Science Society of America Journal, v.60, p. 150-157, 1996.

BRASIL. Ministério de Agricultura. Departamento Nacional de Produção Vegetal. Divisão de Sementes e Mudas. Regras para análise de sementes. Brasília, 1976. 188p.

BRIDGES, C.D.; McGUIRE, J.A.; MARTIN, N.R. Efficiency of chemical and mechanical methods for controlling weeds in peanuts (Arachis hipogea), Weed Science, v.32, p.584$591,1984$.

COSTA, M.P. Efeito da matéria orgânica em alguns atributos do solo. Escola Superior de Agricultura "Luiz de Queiroz" da Universidade de São Paulo, Piracicaba, SP, 1983. (Dissertação de Mestrado). 137 p.

JOHNSON, W.C.; MULLINIX JR, B. G. Phytotoxicity of of flumetsulam on peanut (Arachis hypogaea). Weed Technology, v.10, n.3, p.481-487, 1996.

KHAN, S.U.; BEHKI, R.M. Effects of Pseudomonas species on the release of bound ${ }^{14} \mathrm{C}$ residues from soil treated with $\left[{ }^{14}\right]$ atrazine. Journal of Agricultural and Food Chemistry, v.38, p.2090-2093, 1990.

MONTEIRO, R.T. Degradação de pesticidas. In: MELO, I.S.; AZEVEDO, J.L., eds. Microbiologia ambiental. Jaguariúna: EMBRAPA/CNPDIA, 1997.p.107-124.

NOVO, M.D.D.S.; ORTOLAN, M.C. Spontaneus nodulation, isolation of rhizobia and persistence of herbicides in area of sugar-cane peanut rotations. Revista de Microbiologia, v.22, n.1, p.60-65, 1991. 
PEIXOTO, M.F.S.P.; SAMPAIO, L.S. de V.; PEIXOTO, C.P.; SAMPAIO, H.S. de V.; ABREU, K.C.L.M.; SILVA, T. O. Doses de trifluralina na nodulação e qualidade fisiológica de plantas de soja. Magistra, v.14, n. 1, p.9-17, 2002.

QUEIROZ, S.R. Biodegradação de ${ }^{14} \mathrm{C}$-atrazina em condições semi controladas. Escola Superior de Agricultura "Luiz de Queiroz" da Universidade de São Paulo, Piracicaba, SP, 1983 (Dissertação de Mestrado). 95 p.

REZENDE, P.M.; VIEIRA, M.G.G.C.; NETO, J.C.V.; ALCÃNTARA, E.N. Influência da aplicação de herbicidas no rendimento de grãos, nodulação e qualidade fisiológica de sementes de soja. Pesquisa Agropecuária Brasileira, v.21, n.1, p.25-30, 1985.

RODRIGUES, B.N.; ALMEIDA, F.S.de. Guia de herbicidas. 4 ed. Londrina: 1998.648 p.

SADER, R.; CARVALHO, N.M.; CAMARGO, M. Efeito de diferentes doses de trifluralina (Treflan) no poder germinativo e produção de sementes de amendoim. Revista Brasileira de Sementes, v.1, n.1, p.59-63, 1979.

STOLP, N.B.; SHEA, P.J. Alachlor and atrazine degradation in Nebraska soil and underlying sediments. Soil Science, v.160, n.5, p.359-370, 1995.
VANDERHEYDEN, V.; DEBONGNIE, P.; PUSSEMIER, L. Accelerated degradation and mineralization of atrazine in surface and subsurface soil materials. Pesticide Science, v.49, p.237-242, 1997.

VIDAL, R.A.; FLECK, N.G. Inibidores do crescimento da parte aérea. In: VIDAL, R.A.; MEROTTO Jr., A., eds. Herbicidologia. Porto Alegre: Evangraf, 2001a. p.123-130.

VIDAL, R.A.; FLECK, N.G. Inibidores da polimerizção da tubulina. In: VIDAL, R.A.; MEROTTO Jr., A., eds. Herbicidologia. Porto Alegre: Evangraf, 2001b. p.131137.

WAIS, A.; HAIDER, K.; SPITELLER, M.; GRAAF, A. A.; BURAUEL, P.; FÜHR, F. Using ${ }^{13} \mathrm{C}-\mathrm{NMR}$ spectroscopy to evaluate the binding mechanism of bound pesticide residues in soils. Journal Environmental Science and Health, B22, n.1, p.1-25, 1995.

YASSIR, A.; RIEU, C.; SOULAS, G. Microbial N-dealkylation of atrazine: effect of exogeneaus organic substrates and behaviour of the soil microflora. Pesticide Science, v.54, p.75-82, 1998. 